\title{
Modiolar Dehiscence Associated with Sensorineural Hearing Loss
}

\author{
Jamie P. Treadway ${ }^{\star}$, Joni K. Doherty ${ }^{\star}$, and Fred H. Linthicum Jr. \\ Jamie P. Treadway: jtreadway@hei.org; Fred H. Linthicum: flinthicum@hei.org \\ *House Research Institute; Eccles Temporal Bone Histopathology Laboratory
}

\section{Introduction}

In the normal cochlea, spiral ganglion cell axons merge in the base of the modiolus to form the beginning of the auditory branch of the eighth cranial nerve. The spiral ganglion neuron bodies are located within the spiral (i.e., Rosenthal's) canal of the modiolus, and their central processes converge towards the internal auditory meatus, forming the cochlear nerve (1-3). This temporal bone histopathology case describes displacement of the spiral ganglion neuron cell bodies from Rosenthal's canal into the internal auditory canal. The patient had a moderate sensorineural hearing loss and remarkably poor speech discrimination for her hearing thresholds. We propose that the delay in transmission of signal as well as electrical current leakage contributed to her poor discrimination.

\section{Case Report}

A 70-year-old female reported progressive hearing loss and tinnitus. She had experienced tinnitus for 25 years at the time of initial evaluation. She had no family history of hearing loss, otitis media, or vertigo. She had worn a hearing aid in her left ear for the previous ten years. An audiogram revealed moderate sensorineural hearing loss in both ears in the frequency range of $500 \mathrm{~Hz}$ to $4000 \mathrm{~Hz}$, with slightly better $(\sim 10 \mathrm{~dB})$ thresholds in the right ear across the frequency spectrum (Figure 1). Further, there was a downsloping of thresholds from $4000 \mathrm{~Hz}$ to $6000 \mathrm{~Hz}$ in both ears. The patient's speech reception thresholds (SRT) were $50 \mathrm{~dB}$ in the right ear (AD) and $60 \mathrm{~dB}$ in the left ear (AS). However, her word discrimination was poor at $36 \% \mathrm{AD}$ and $48 \%$ AS. The patient's last audiogram was performed fourteen years prior to her death in 1974.

\section{Histopathology}

The right temporal bone shows some atrophy of the striae (30\%), and only about $1 / 3$ of the usual number of neurons and ganglion cells were found in the modiolus. The auditory nerve has been avulsed during harvesting on the right.

Corresponding author: Joni K. Doherty, MD, PhD, FACS, Co-Director, Eccles Temporal Bone Histopathology Laboratory, House Research Institute, 2100 West third street, Los Angeles, California 90057, Phone 213-273-8031, Fax 213-413-6739, jdoherty@ hei.org. 
The left temporal bone shows $25 \%$ atrophy of the striae. The Organ of Corti is absent in the basal turn, and there are no neurites in the osseous spiral lamina. The Organ of Corti is deformed in the rest of the turns, and there are only 1/10 the number of spiral ganglion neurons normally present in the modiolus. Ganglion cells were found scattered throughout the auditory nerve as far as one millimeter medial to its exit from the cochlea (Figure 2). We used stereology with oil immersion microscopy to measure the distance from the hair cell to ganglion cell body in this case compared with that in normal cochlea. This distance of neuritis to the spiral ganglion neurons varies in this case, but is, on average, approximately three times the normal distance from hair cells to the spiral ganglion cell body within the modiolus. We propose that the increased distance would result in delayed transmission of sound sensation to the cochlear nerve, since nerve transmission via peripheral processes (i.e., dendrites) is slower compared with axonal transmission (4). This has been shown in modeling of dendrites, and is due to the increased resistance on electrical transmission due to the narrow diameter of dendrites compared with axons (5). Additionally, increased length of neuronal dendrites promotes greater 'leakage' of the electrical current (4). Although some cases of limited modiolar dehiscence with some spiral ganglion cell exposure have been reported, to our knowledge, this is the first instance of widespread spiral ganglion cell departure from the base of the modiolus with presence relatively deep into the auditory nerve within the internal auditory canal (IAC).

\section{Discussion}

This patient had unexpectedly low speech discrimination for her pure tone thresholds and SRT. Some of this was undoubtedly attributable to a deformed Organ of Corti with hair cell and supporting cell loss, lack of a complete stria vascularis, and the low spiral ganglion cell counts. Additionally, the unusual finding of cochlear neuron cell bodies within the IAC portion of the auditory nerve suggests that there would be a significant delay in transduced sound potentials reaching the spiral ganglion cell bodies in this location, since it is much further from the normal location of SGN within the modiolus. Therefore, we feel that this anatomical abnormality contributed to her poor hearing.

\section{References}

1. Tuncel M, Sürücü HS, Erbil KM, Konan A, et al. Formation of the Cochlear Nerve in the Modiolus of the Guinea Pig and Human Cochleae. Archives of Medical Research. 2005; 36:436-440. [PubMed: 16099318]

2. Spoendlin H. Anatomy of cochlear innervation. Am J Otolaryngol. 1985; 6:453-67. [PubMed: 3909832]

3. Defourny J, Lallemand F, Malgrange B. Structure and development of cochlear afferent innervation in mammals. Am J Physiol Cell Physiol. 2011; 301:C750-61. [PubMed: 21753183]

4. Korogod SM, Kaspirzhny AV. Spatial heterogeneity of passive electrical transfer properties of neuronal dendrites due to their metrical asymmetry. Biol Cybern. 2011; 105:305-17. [PubMed: 22215007]

5. Nadol JB. Comparative anatomy of the cochlea and auditory nerve in mammals. Hearing Research. 1988; 34:253-66. [PubMed: 3049492] 


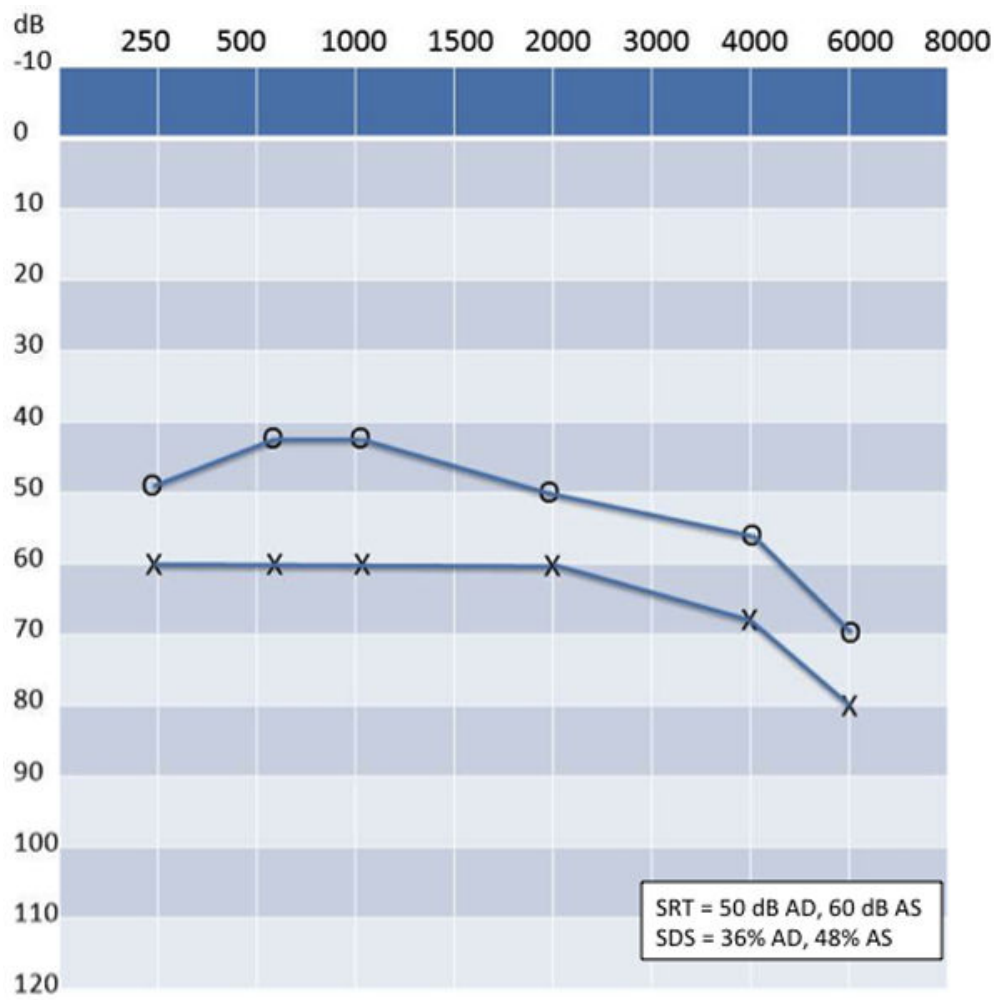

Figure 1.

Patient's audiogram, demonstrating moderate sensorineural hearing loss and poor word discrimination. 


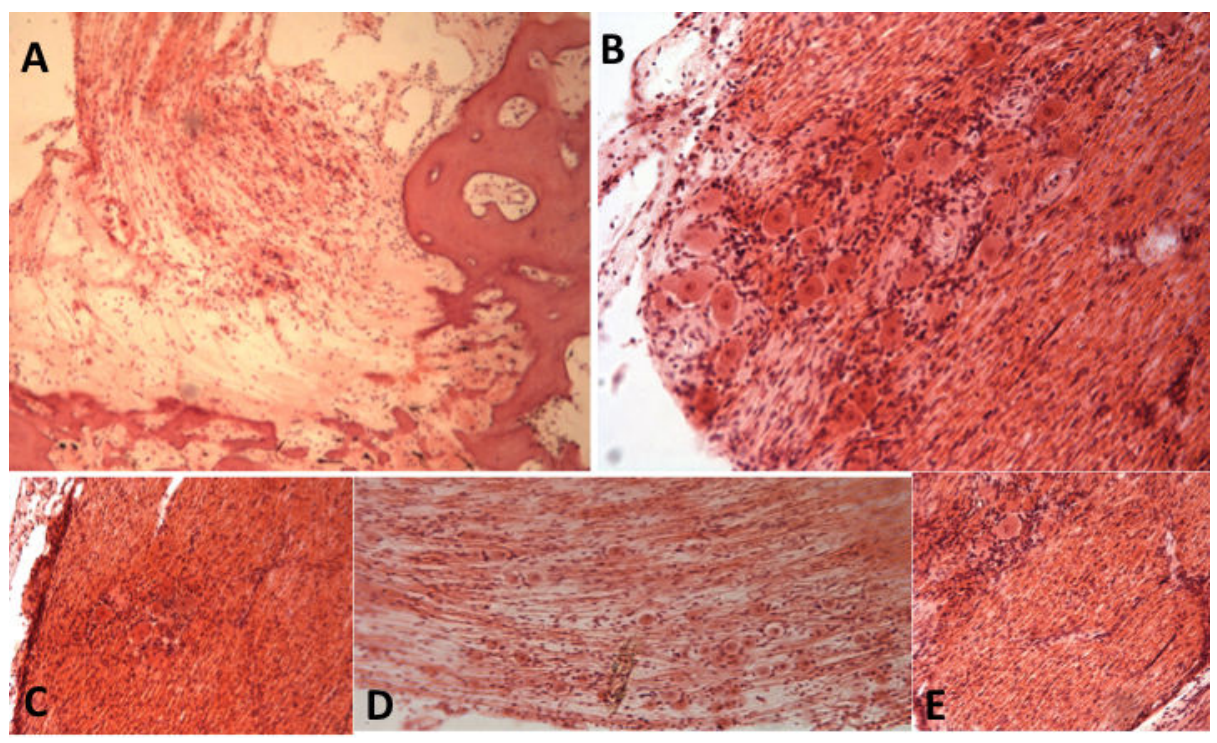

Figure 2.

Histopathological sections (celloidin embedded, $20 \mu \mathrm{m}$ ) from this patient's left temporal bone stained with hematoxylin and eosin (H\&E): at the base of the cochlea where the base of the modiolus displays dehiscence (A), and spiral ganglion neurons are visible within the internal auditory canal portion of the auditory nerve (B-E). 